\title{
Exciton-Polariton Gap Solitons in Two-Dimensional Lattices
}

\author{
E. A. Cerda-Méndez, ${ }^{1, *}$ D. Sarkar, ${ }^{2, \dagger}$ D. N. Krizhanovskii, ${ }^{2}$ S. S. Gavrilov, ${ }^{3}$ K. Biermann, ${ }^{1}$ \\ M. S. Skolnick, ${ }^{2}$ and P. V. Santos ${ }^{1}$ \\ ${ }^{1}$ Paul-Drude-Institut für Festkörperelektronik, 10117 Berlin, Germany \\ ${ }^{2}$ University of Sheffield, Sheffield S37RH, United Kingdom \\ ${ }^{3}$ Institute of Solid State Physics, Chernogolovka 142432, Russia \\ (Received 23 May 2013; revised manuscript received 16 August 2013; published 2 October 2013)
}

We report on the two-dimensional gap-soliton nature of exciton-polariton macroscopic coherent phases (PMCP) in a square lattice with a tunable amplitude. The resonantly excited PMCP forms close to the negative mass $M$ point of the lattice band structure with energy within the lattice band gap and its wave function localized within a few lattice periods. The PMCPs are well described as gap solitons resulting from the interplay between repulsive polariton-polariton interactions and effective attractive forces due to the negative mass. The solitonic nature accounts for the reduction of the PMCP coherence length and optical excitation threshold with increasing lattice amplitude.

The periodic spatial modulation of a medium creates an artificial band structure with energy gaps and anomalous (i.e., negative) dispersion. In the presence of nonlinearity, spatially self-localized states may appear within the energy gaps as the result of the interplay between the anomalous dispersion and interparticle interactions. This takes place when the kinetic energy contribution $\left[E_{K}=\right.$ $\left.-\pi^{2} \hbar^{2} /\left(2 m_{b} \rho^{2}\right)\right]$ due to localization of particles with a negative mass $-m_{b}$ within a radius $\rho$ compensates the repulsive interparticle interaction energy $E_{I}$. These states, known as gap solitons (GSs), are metastable solutions of the Gross-Pitaevskii equation [1]. GSs have been explored in optical fibers [2], nonlinear photonic crystals [3-6], atomic Bose Einstein condensates (BECs) in optical lattices $[7,8]$, and, very recently, also in the hybrid lightmatter polariton system [9]. Polaritons result from the strong coupling of photons and quantum well (QW) excitons in a semiconductor microcavity (MC). Being bosonic light-matter quasiparticles, they advantageously combine features from both species. Namely, the small mass arising from the photonic component allows them to form polariton macroscopic coherent phases (PMCPs) at low densities and high temperatures, while the interexcitonic interactions provide a nonlinearity several orders of magnitude stronger than in purely photonic systems [10]. While GSs in one-dimensional (1D) potentials have been extensively studied [2,3,5,7-9], GSs in 2D lattices have so far only been reported for purely photonic systems $[4,6]$. GSs in 2D potentials are qualitatively different from their 1D counterparts, for example, opening the way to the realization of novel topological phases $[6,11]$.

In this Letter, we demonstrate the formation and manipulation of GSs of PMCPs in a 2D tunable lattice. The studies were carried out in PMCPs resonantly excited in a tunable square lattice created by surface acoustic waves (SAWs). While PMCPs in a homogeneous MC normally appear at the lowest energy state with zero in-plane momentum, PMCPs in a shallow (i.e., low amplitude) lattice have a GS character and are excited via the accumulation of particles at critical points of negative mass and energy above the ground state [12]. The PMCP forms close to the negative effective mass $M$ states of the lattice band structure, its energy lies within the band gap and its wave function is localized within a few lattice periods, i.e., over a region smaller than the optical excitation area. By taking advantage of the tunability of the acoustic lattice, we show that increasing the lattice amplitude reduces the coherence length $L_{\text {coh }}$ as well as the optical threshold power $\left(P_{\text {th }}\right)$ for the excitation of the PMCP. These properties are well described using a simple analytical energetic model $[7,13]$.

The sample is an (Al,Ga)As-based MC [14] where a $170 \times 170 \mu \mathrm{m}^{2}$ sinusoidal square lattice is created by interfering two SAWs [Fig. 1(a)]. The MC consists of two Bragg reflectors [stacks of $(\mathrm{Al}, \mathrm{Ga}) \mathrm{As} \lambda / 4$ layers with a different index of refraction] embedding a $\lambda / 2$ cavity with three pairs of $15 \mathrm{~nm}$-thick GaAs QWs ( $\lambda$ is the wavelength of the confined photon). SAWs with wavelength $\lambda_{\text {SAW }}=$ $8 \mu \mathrm{m}$, frequency of $370 \mathrm{MHz}$, and propagation velocity of $3 \mu \mathrm{m} / \mathrm{ns}$ were excited by acoustic transducers deposited on the sample surface. The formation of the lattice results from the modulation of the excitonic band gap and the microcavity optical resonance by the SAW strain field [14]. The polaritons were resonantly excited within a $70 \mu \mathrm{m}$ spot using a single-mode, continuous-wave Gaussian pump laser delivering photons with energy $E_{\text {pump }}=1.5353 \mathrm{meV}$ and in-plane momentum $\mathbf{k}_{\text {pump }}=$ $\left(k_{\mathrm{p}_{\mathrm{x}}}, k_{\mathrm{p}_{\mathrm{y}}}\right)=(0,1.7) \mu \mathrm{m}^{-1}$. The studies were performed by recording the steady-state photoluminescence (PL) emerging from the sample top surface (time integration of a few seconds) with spatial and angular (i.e., $k$ ) resolution.

The square lattice creates mini-Brillouin zones (MBZs) of dimension $k_{\mathrm{SAW}}=2 \pi / \lambda_{\mathrm{SAW}}$ separated by energy gaps 

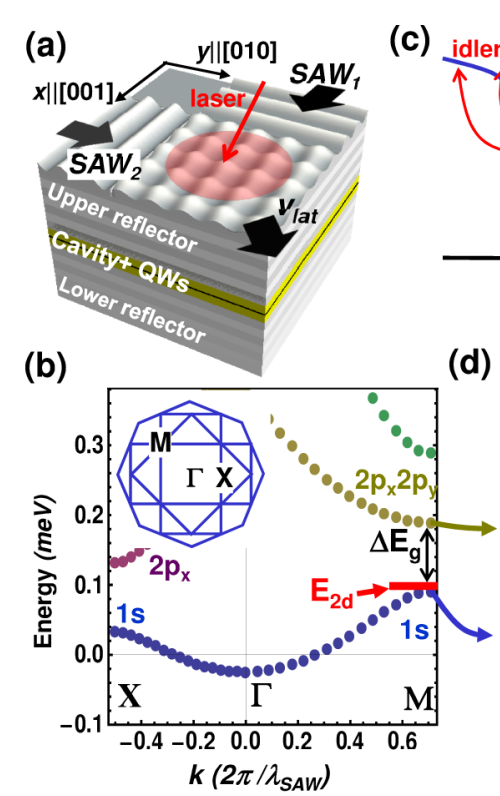

(d)
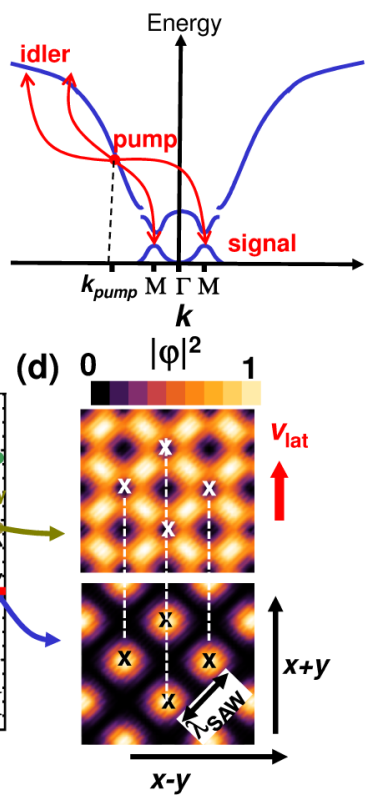

FIG. 1 (color online). (a) Square lattice for polaritons created by the interference of two surface acoustic waves (SAWs) propagating along the $\hat{x}=[010]$ and $\hat{y}=[001]$ surface directions of a (100)-(Al,Ga)As MC. The lattice moves along the $(\hat{x}+\hat{y})=[110]$ direction with a velocity of $v_{\text {lat }}=\sqrt{2} v_{\mathrm{SAW}}$, where $v_{\mathrm{SAW}}=3 \mu \mathrm{m} / \mathrm{ns}$ is the SAW phase velocity. (b) Oneparticle band structure for polaritons in a shallow lattice $\left(\Phi_{\mathrm{SAW}}=50 \mu \mathrm{eV}\right) . E_{2 d}$ denotes the soliton energy and wave vector range. The energy is relative to the bottom of the unmodulated dispersion. The inset is a diagram of the first four mini-Brillouin zones of the square lattice. (c) Schematic representation of the OPO process in the modulated polariton dispersion along $\Gamma \rightarrow M$. The pump state is generated by tuning the laser energy and angle of incidence. Above the threshold of formation of the PMCP polaritons scatter into the signal and idler states, as indicated by the arrows. (d) Calculated squared moduli of the wave function $|\psi|^{2}$ of the $1 s$ and $2 p_{\mathrm{x}} 2 p_{\mathrm{y}}$ states at the $M$ point. The crosses mark the minima of the potential lattice. The whole pattern moves along the vertical direction with velocity $v_{\text {lat }}$.

[cf. inset Fig. 1(b)] [15]. Figure 1(b) displays the band diagram along the $X \rightarrow \Gamma \rightarrow M$ direction calculated for a shallow lattice, where the curvature of the $s$ band inverts close to $X$ and $M$. In the shallow lattice, where the modulation amplitude $2 \Phi_{\mathrm{SAW}}=0.1 \mathrm{meV}$ is smaller than the energetic width of the lowest branch $\left(\hbar k_{\mathrm{SAW}}\right)^{2} / 2 m_{p} \approx$ $0.4 \mathrm{meV}\left(m_{p}=6 \times 10^{-5} m_{\mathrm{e}}\right.$ and $m_{\mathrm{e}}$ are the polariton mass at $\Gamma$ and free electron mass, respectively) $X$ is a saddle point with positive mass $m_{p}$ along $X \rightarrow M$ and negative mass $-m_{b}=-0.5\left(m_{p} \lambda_{\mathrm{SAW}} / \pi \hbar\right)^{2} \Delta E_{\mathrm{g}} \quad$ along $\quad X \rightarrow \Gamma \quad$ [see Supplemental Material (SM), Sec. I, [16]]. Here, $\Delta E_{g} \approx$ $2 \Phi_{\text {SAW }}$ is the energy gap between the first and second folded bands [14]. $M$, in contrast, has a negative effective mass equal to $-m_{\mathrm{b}}$ along both $M \rightarrow \Gamma$ and $M \rightarrow X$. $\Phi_{\mathrm{SAW}}$ can be controlled by the radio-frequency power $P_{\mathrm{rf}}$ applied to the acoustic transducers since $\Phi_{\mathrm{SAW}} \propto \sqrt{P_{\mathrm{rf}}}$ [14].

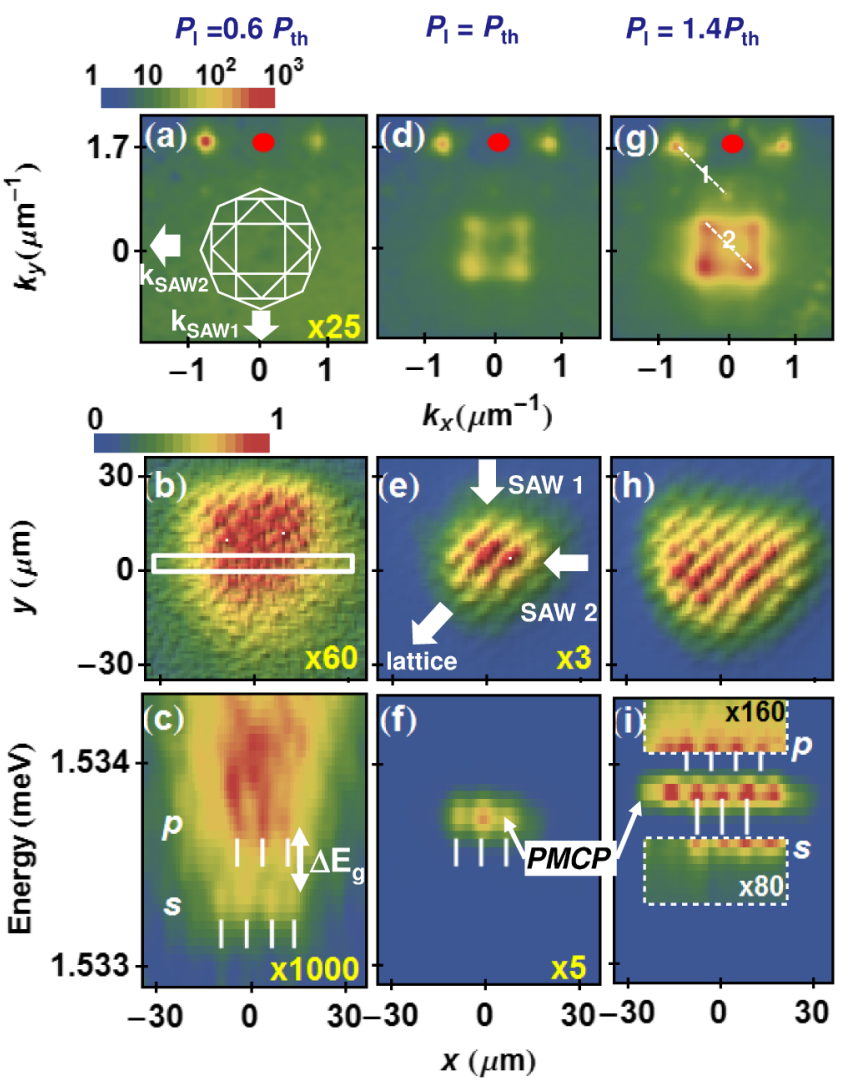

FIG. 2 (color online). (a) $k$-space PL image of an incoherent polariton gas. The red point at $\mathbf{k}_{\mathbf{p}}=\left(k_{\mathrm{p}_{\mathrm{x}}}, k_{\mathrm{p}_{\mathrm{y}}}\right)=(0,1.7) \mu \mathrm{m}^{-1}$ marks the pump state, which was blocked during the experiment. The PL peaks at $\mathbf{k}=\left( \pm k_{\mathrm{SAW}}, k_{\mathrm{p}_{\mathrm{y}}}\right)=(0.78,1.7) \mu \mathrm{m}^{-1}$ and at $\mathbf{k}=\left( \pm k_{\mathrm{SAW}}, k_{\mathrm{p}_{\mathrm{y}}}-k_{\mathrm{SAW}}\right)=(0.78,0.9) \mu \mathrm{m}^{-1}$ are the diffracted pump beams. The white lines delineate the first four MBZs. (b) Real-space PL image of an incoherent polariton gas at a pump power $P_{\ell}<P_{\text {th }}$. (c) Spatially resolved spectrum along the slit in (b). The dotted lines mark the positions of the lattice $s$ and $p$ states. (d)-(f) and (g)-(i) are the corresponding images at $P_{\ell}=P_{\text {th }}$ and at $P_{\ell}=1.4 P_{\text {th. }}$. The image intensities were amplified within the boxes in (i). In all cases, $P_{\mathrm{rf}}=22 \mathrm{~mW}$.

The lattice emission properties below $P_{\text {th }}$ are summarized in the leftmost panels of Fig. 2. The $k$-space image of Fig. 2(a) is dominated by the bright spots around $\mathbf{k}_{\text {pump }}$, which are the diffracted replicas of the pump polariton state. Since this image is not energy resolved, the emission around $\mathbf{k}=(0,0)$ appears as a weak and unstructured background. The white lines delineate, for reference, the contours of the first four MBZs, as in Fig. 1(b).

The real-space emission map in Fig. 2(b) shows an intensity distribution reflecting the Gaussian shape of the pump laser beam. Finally, Fig. 2(c) displays the PL spectral distribution along the slit delineated in panel (b). The broad energy range of the emission indicates that the level population below $P_{\text {th }}$ essentially reflects the polariton density of states and is mainly governed by incoherent (multistep) energy relaxation rather than by direct scattering of particles from the pump states. 
A closer examination of Fig. 2(b) also reveals faint diagonal lines, which trace the paths of the moving sites of the lattice [cf. Fig. 1(a)] [15]. The PL from the lowest energy bonding $(s)$ and anti-bonding $(p)$ lattice states in Fig. 2(c) are spatially shifted (white lines) in agreement with the calculations in Fig. 1(d). The latter shows that the maxima of the $s$ and $p$ wave functions follow separated paths [17].

For higher excitation intensities, a PMCP forms via an optical parametric oscillator (OPO) process [18] depicted in Fig. 1(c). Here, polaritons in the pump state scatter into states with a lower (signal) and a higher (idler) energy conserving energy $\left[2 E_{\text {pump }}=E_{\text {signal }}+E_{\text {idler }}\right]$ and in-plane wave vector [ $\left.2 k_{\text {pump }}=k_{\text {signal }}+k_{\text {idler }}\right]$. Interestingly, the experimental $k$-space map of Fig. 2(d) shows that the signal $\mathrm{PMCP}$ in a shallow square lattice forms at the negative mass $M$ points [i.e., $\left.\mathbf{k}_{\text {signal }}=( \pm 0.5, \pm 0.5) k_{\mathrm{SAW}}\right)$, cf. Fig. 1(c)]. Further results included in the Supplemental Material (Sec. III) [16] prove that the formation of a PMCP at $M$ is insensitive to the orientation of $\mathbf{k}_{\text {pump }}$ with respect to the lattice and that all the $M$-point peaks emit at the same energy within the experimental spectral resolution of $60 \mu \mathrm{eV}$. The real space map of Fig. 2(e) indicates that the PMCP emission extends over approximately three central lattice sites with an intensity orders of magnitude stronger than below $P_{\text {th }}$. Finally, the spectrum in panel (f) shows the energy blueshift and linewidth reduction characteristic of the formation of a PMCP.

The OPO stimulated scattering requires a critical density $N_{2 \mathrm{D} \text {,min }}$ of signal particles. Since no selective scattering takes place below $P_{\text {th }}$, the preferential accumulation of particles at $M$ is attributed to its anomalous (negative) dispersion. This behavior contrasts to PMCPs in unmodulated MCs, where $N_{2 \mathrm{D} \text {, min }}$ is typically achieved by particle accumulation close to the minimum of the dispersion [i.e., $\left.\mathbf{k}_{\text {signal }}=(0,0)\right]$.

In order to determine the energy of the $\mathrm{PMCP}$ relative to the $s$ and $p$ band states, we have increased $P_{\ell}$ to $\approx 1.4 P_{\text {th }}$ to enhance the residual emission from these states. The amplified insets of the energy region below and above the PMCP [boxes in Fig. 2(i)] reveal that the emission peaks of the PMCP are aligned with the positions of the $s$ states and displaced with respect to the $p$ states. Although the exact PMCP emission energy cannot be determined due to the limited spectral resolution, Fig. 2(i) shows that it appears above of the $s$ and below the $p$ bands.

So far, we have shown that the PMCP forms close to the negative-mass $M$ states of the lowest energy band. Another important feature is the limited size of the PMCP wave function, which is quantified by the spatial coherence length $L_{\text {coh }}$ of the PL. $L_{\text {coh }}$ can be extracted from the $k$-space maps in Fig. 2 by using Heisenberg's uncertainty relationship $\Delta x \Delta k \geq 2 \pi$, where $\Delta x=L_{\mathrm{coh}}$ and $\Delta k$ is the full width at half maximum (FWHM) of the emission peaks. At threshold [cf. Fig. 2(d)], $L_{\text {coh }} \approx 27 \mu \mathrm{m} \sim 3 \lambda_{\text {SAW }}$ coincides with the diameter of the real-space image in Fig. 2(e). A $40 \%$ increase in $P_{\ell}$ doubles the PMCP diameter [Fig. 2(h)], but changes only slightly the FWHM of the diffraction peaks [cf. Fig. 2(g)], thus indicating that $L_{\text {coh }}$ is essentially independent of the total size of the PMCP.

While the preferential accumulation of particles at $M$ results from the negative mass, the formation of a PMCP with a limited $L_{\text {coh }}$ is attributed to the excitation of a GS in the shallow lattice. The GS consist of a superposition of $M$ states with an energy $E_{2 d}$ within the band gap and close to the top of the $s$ band. The observation of a large emission area ( $\gg L_{\text {coh }}$ ) for light fluxes above threshold [cf. Fig. 2(h)] can thus be accounted for by the excitation of several GSs with similar $L_{\text {coh }}$ at random positions of the potential.

The tunability of the potential provides a powerful tool to investigate the dependence of the GS properties, in particular those related to self-localization, on the lattice amplitude $\Phi_{\mathrm{SAW}}$. Figure 3(a) compares $k$-space intensity profiles along $M \rightarrow \Gamma \rightarrow M$ for the pump and signal PMCP states recorded along the dashed lines in Fig. 2(g). The coordinate $k_{0}$ of the peaks in the pump profile (topmost line) defines the limits of the 1st MBZ and does not change with $\Phi_{\mathrm{SAW}}$. The GS peaks (lower lines), however, broaden, and their maxima $k_{0}$ move inwards within the MBZ. Both features are ascribed to the self-localization of the GS wave packet, as discussed in detail below. Since $k_{0}$ for the pump replicas remains fixed for all $\Phi_{\mathrm{SAW}}$, we conclude that the $k$-space configuration of the GS is mainly determined by its particle density and by $\Phi_{\mathrm{SAW}}$, rather than by interactions with the pump states.

It is notable that increasing $\Phi_{\mathrm{SAW}}$ also reduces the optical threshold $P_{\text {th }}$ for GS excitation, as indicated by
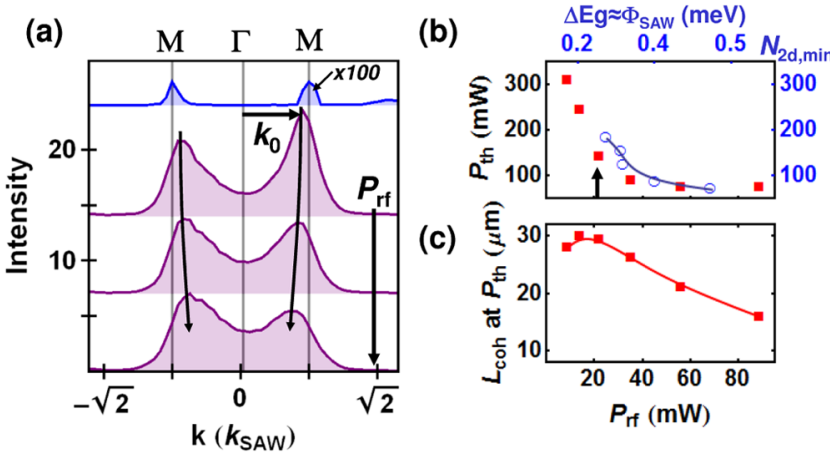

FIG. 3 (color online). (a) Intensity profiles along a $M \rightarrow \Gamma \rightarrow M$ direction [cf. dashed lines 1 and 2 in Fig. 2(f)]. The topmost curve for the diffracted pump beam (line 1) defines the limits of the first MBZ. The additional curves are profiles for the signal PMCP (line 2) in lattices created with $P_{\mathrm{rf}}=22,35$, and $56 \mathrm{~mW}$ (from the second to the bottom curves). (b) Experimental dependence of $P_{\text {th }}$ on $P_{\text {rf }}$ (squares). The empty circles show the calculated dependence of the minimum number of polaritons $N_{2 d \text {, min }}$ to form the soliton with $\Phi_{\text {SAW }}$. The line is a guide to the eye. The parameters used are listed in the caption of Fig. 4. (c) Dependence of the PMCP coherence length $\left(L_{\mathrm{coh}}\right)$ at threshold on $P_{\mathrm{rf}}$. 
the squares in Fig. 3(b). This behavior can be understood by setting $E_{I}\left(N_{2 d}\right)=N_{2 d} g_{2 d} / \rho^{2}$ in the equality $E_{\mathrm{I}}\left(N_{2 d}\right)=E_{K}$ defined in the introduction, where $N_{2 d}$ is the particle number and $g_{2 d}>0$ their characteristic interaction constant. The latter yields a number of particles in a stable GS of $N_{2 d}=$ $\pi^{2} \hbar^{2} /\left(2 g_{2 d} m_{b}\right)$. Since $\left|m_{b}\right|$ increases with $\Phi_{\text {SAW }}$ (see SM, Sec. I [16]), $N_{2 d}$, and consequently $P_{\text {th }}$, must decrease. Finally, Fig. 3(c) shows that the reduction in $P_{\text {th }}$ with $P_{\mathrm{rf}}$ is accompanied by a decrease of the value of $L_{\text {coh }}$ at threshold.

In order to quantitatively support the GS description we have used a variational approach [13] to calculate metastable states in a square lattice defined by a potential $V(x, y)=$ $-\Phi_{\mathrm{SAW}}\left[\cos \left(k_{\mathrm{SAW}} x\right)+\cos \left(k_{\mathrm{SAW}} y\right)\right]$. This approach has been shown to be compatible with numerical solutions of the Gross-Pitaevskii equation for BECs out of equilibrium [7]. Despite the fact that the OPO is a nonequilibrium threestate system, this simple approach gives a consistent physical picture of the main experimental findings by describing only the energetic configuration of the signal PMCP state. The results are also well reproduced by full numerical solution of the Gross-Pitaevskii equation (see Supplemental Material, Sec. II [16]). We assume a trial wave function consisting of Bloch states of the square lattice with an envelope function given by

$\Psi_{2 d}(x, y)=\sqrt{\frac{N_{2 d}}{\pi r_{0}^{2}}} \frac{2 e^{-\left(x^{2}+y^{2}\right) /\left(2 r_{0}^{2}\right)}}{\left(e^{-\left(k_{0} r_{0}\right)^{2}}+1\right)} \cos \left(k_{0} x\right) \cos \left(k_{0} y\right)$.

This expression includes, in addition to the Gaussian term with width $r_{0}$ proposed in Ref. [13], two oscillating cosine terms with wave vector $k_{0} . r_{0}$ is related to the coherence length $L_{\mathrm{coh}}$ of the state by $L_{\mathrm{coh}}=\sqrt{2 \ln 2} r_{0}$.

Figure 4(a) displays the energy for the state $\Psi_{2 d}$ as a function of the parameters $r_{0}$ and $k_{0}$ calculated for a fixed number of particles $N_{2 d}$. The ground state corresponds to the extended (s) band state at $k_{0}=0$ and $r_{0} \rightarrow \infty$ (out of the scale of the plot). The potential landscape also exhibits a valley indicated by the dashed line. For particular combinations of $N_{2 d}$ and $\Phi_{\mathrm{SAW}}$, local minima $\left(M_{1}\right.$ and $\left.M_{2}\right)$ may appear within this valley. These minima correspond to GS states with coherence length $L_{\text {coh }}$, effective wave vector $k_{0}$, and energy within the lattice band gap $\Delta E_{g}$ [cf. Fig. 1(c)]. While $M_{1}$ is a GS localized within a single unit cell, $M_{2}$ is an extended mode spreading over several lattice unit cells (see Supplemental Material, Sec. IV [16]). The position of the minima changes with $N_{2 d}$ and/or $\Phi_{\text {SAW }}$.

The PMCP at the $M$ point reported in this work is ascribed to an $M_{2}$ GS mode with $k_{0}<k_{\mathrm{SAW}} / 2=$ $\pi / \lambda_{\mathrm{SAW}}$ and $L_{\mathrm{coh}}$ of a few SAW periods. The open circles in Fig. 4(b) display the dependence of $k_{0}$ of $M_{2}$ on $L_{\mathrm{coh}}$ (open circles) calculated for a fixed value of $N_{2 d}$ while varying $\Phi_{\text {SAW }}$. The squares display the corresponding experimental values obtained from Fig. 3(c) (i.e., $k_{0}$ vs $\left.L_{\mathrm{coh}}\right)$. The calculations reproduce well the observed
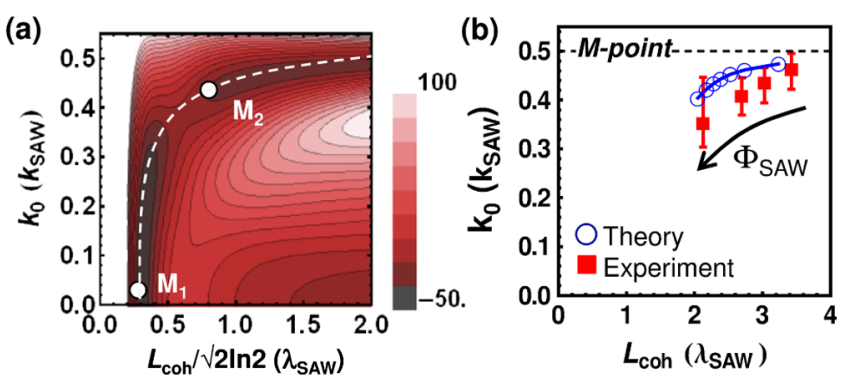

FIG. 4 (color online). (a) Energy map of the state defined by Eq. (1) (color scale) as a function of parameters $r_{0}$ and $k_{0}$ calculated for $N_{2 \mathrm{~d}}=150$ polaritons, $\Phi_{\mathrm{SAW}}=200 \mu \mathrm{eV}, m_{p}=$ $6 \times 10^{-5} m_{e}, \lambda_{\mathrm{SAW}}=8 \mu \mathrm{m}$, and particle interaction constant $g_{2 d}=10 \mu \mathrm{eV} \mu \mathrm{m}^{2} . M_{1}$ and $M_{2}$ mark local energy minima corresponding to GS solutions. The white line is a guide to the eye. (b) Experimental (squares) and calculated (circles) dependence of the $k$-coordinates of the maxima of the emission of peaks $k_{0}$ on $L_{\text {coh }}$. The red squares are the experimental points obtained for $P_{\ell}=1.2 P_{\text {th }}$ and the open circles are the calculated points.

displacement of the PMCP $k$-space peaks with $\Phi_{\mathrm{SAW}}$. Physically, the reduction of $k_{0}$ with $L_{\text {coh }}$ is analogous to the downwards shift in frequency of a harmonic oscillator with increasing damping. Finally, the model can also qualitatively account for the observed reduction of $P_{\text {th }}$ with increasing $P_{\text {rf }}$ displayed in Fig. 3(b). Here, the circles show that the calculated minimum particle number $N_{2 d \text {,min }}$ required for the formation of the $M_{2}$ states reduces as $\Phi_{\text {SAW }}$ increases.

In conclusion, we have presented experimental evidence for the gap solitonic nature of PMCPs resonantly excited in a 2D shallow tunable lattice. The soliton PMCP forms at a critical point of negative mass due to the combined effects of particle attraction and repulsive interparticle interactions. This work opens the way for the study of lattice vortex solitons and other polariton quantum phases such as a Bose glass.

The authors wish to thank L. Schrottke and D. Skryabin for fruitful discussions and A. Tahraoui, S. Rauwerdink, W. Seidel, and B. Drescher for sample processing. This work was supported by ESF-POLATOM Grants No. 5111 and No. 5369, EPSRC Grants No. EP/G001642 and No. EP/ J007544, DFG Grant No. CE 191/2-1, EU ITN Clermont 4 Grant and RFBR Grant No. 13-02-12139.

*ecerda@pdi-berlin.de

†Present address: Universidad Autónoma de Madrid, Madrid, Spain.

[1] Y. V. Kartashov, B. A. Malomed, and L. Torner, Rev. Mod. Phys. 83, 247 (2011).

[2] B. J. Eggleton, R. E. Slusher, C. M. de Sterke, P. A. Krug, and J. E. Sipe, Phys. Rev. Lett. 76, 1627 (1996).

[3] W. Chen and D. L. Mills, Phys. Rev. Lett. 58, 160 (1987).

[4] J. W. Fleischer, M. Segev, N. K. Efremidis, and D. N. Christodoulides, Nature (London) 422, 147 (2003). 
[5] D. Neshev, E. Ostrovskaya, Y. Kivshar, and W. Krolikowski, Opt. Lett. 28, 710 (2003).

[6] D. N. Neshev, T. J. Alexander, E. A. Ostrovskaya, Y.S. Kivshar, H. Martin, I. Makasyuk, and Z. Chen, Phys. Rev. Lett. 92, 123903 (2004).

[7] E. A. Ostrovskaya and Y. S. Kivshar, Phys. Rev. Lett. 90, 160407 (2003).

[8] B. Eiermann, T. Anker, M. Albiez, M. Taglieber, P. Treutlein, K.-P. Marzlin, and M. K. Oberthaler, Phys. Rev. Lett. 92, 230401 (2004).

[9] D. Tanese, H. Flayac, D. Solnyshkov, A. Amo, A. Lemaître, E. Galopin, R. Braive, P. Senellart, I. Sagnes, G. Malpuech, and J. Bloch, Nat. Commun. 4, 1749 (2013).

[10] D. M. Whittaker, Phys. Rev. B 71, 115301 (2005).

[11] R. Driben and B. A. Malomed, Eur. Phys. J. D 50, 317 (2008).

[12] A. Gorbach, B. Malomed, and D. Skryabin, Phys. Lett. A 373, 3024 (2009).

[13] B. B. Baizakov, B. A. Malomed, and M. Salerno, Europhys. Lett. 63, 642 (2003).
[14] E. A. Cerda-Méndez, D. N. Krizhanovskii, M. Wouters, R. Bradley, K. Biermann, K. Guda, R. Hey, P. V. Santos, D. Sarkar, and M. S. Skolnick, Phys. Rev. Lett. 105, 116402 (2010).

[15] E. A. Cerda-Méndez, D. N. Krizhanovskii, K. Biermann, R. Hey, M. S. Skolnick, and P. V. Santos, Phys. Rev. B 86, 100301 (2012).

[16] See Supplemental Material at http://link.aps.org/ supplemental/10.1103/PhysRevLett.111.146401 for details. Here, the calculation for the expression for the effective mass at the band edges $m_{b}$, the numerical solution of the Gross-Pitaevskii equation, the experimental results for excitation with different pump orientation and the calculations of the gap soliton wave functions are shown.

[17] Although $|\psi|^{2}$ has been calculated at $M$, the profiles are essentially the same over the whole branch.

[18] R. M. Stevenson, V. N. Astratov, M. S. Skolnick, D. M. Whittaker, M. Emam-Ismail, A. I. Tartakovskii, P. G. Savvidis, J. J. Baumberg, and J. S. Roberts, Phys. Rev. Lett. 85, 3680 (2000). 